\title{
Produção científica e contexto do Mestrado em Ciências do Envelhecimento da Universidade São Judas Tadeu
}

\section{Scientific production and context of Master on Sciences of Aging at São Judas University}

\author{
Ana Lúcia Gattia, Carla Witter ${ }^{b}$, Claudia Aranha Gilc, Rita Maria Monteiro Goulartd* \\ a Doutora, Programa Stricto Sensu de Mestrado em Ciências do Envelhecimento da Universidade São Judas Tadeu - USJT. <algatti@ig.com.br> \\ b Doutora, Programa Stricto Sensu de Mestrado em Ciências do Envelhecimento da Universidade São Judas Tadeu - USJT. <cwitter12@gmail.com> \\ Doutora, Programa Stricto Sensu de Mestrado em Ciências do Envelhecimento da Universidade São Judas Tadeu - USJT. < claudiaagil@uol.com.br> \\ d Doutora, Programa Stricto Sensu de Mestrado em Ciências do Envelhecimento da Universidade São Judas Tadeu - USJT. < ritagoulartnutri@gmail.com>
}

\section{ARTICLE INFO}

\section{Article history}

Received: 02/06/2015

Accepted: 06/07/2015

\section{Correspondent Author}

Carla Witter

Av. Pedroso de Moraes, 144/302

05420-000 São Paulo, SP, Brasil

<cwitter12@gmail.com>

\section{(C) 2014 All rights reserved}

Editors

Alfredo Cataldo Neto

Irenio Gomes

\begin{abstract}
RESUMO
A abordagem integral das questões relacionadas aos idosos requer produção de conhecimento e trabalho especializado. O estabelecimento de programas de pósgraduação voltados para a área da gerontologia e geriatria é a resposta que instituições de ensino têm oferecido. $O$ presente artigo objetiva apresentar o que tem sido produzido no Mestrado em Ciências do Envelhecimento da Universidade São Judas Tadeu, em São Paulo. Os relatórios anuais da Capes, no período entre 2010 e 2014, foram o material sobre o qual foram levantadas, tabuladas e analisadas, de modo independente por duas docentes do curso, as produções intelectuais compreendendo artigos completos e resumos publicados em revistas científicas, anais de eventos nacionais e internacionais da área de envelhecimento e áreas correlatas. Um total de 74 publicações em periódicos, 280 produções em eventos nacionais e 57 em eventos internacionais, em sua maioria realizada em coautoria, por pesquisadores de diversas áreas e níveis de especialização, incluindo a contribuição de profissionais externos à instituição, distribui-se de modo equilibrado ao longo deste período. Observa-se uma disparidade quanto ao número de projetos e pesquisadores quando são consideradas as duas linhas de pesquisa propostas pelo programa, com maior participação da linha que trata dos aspectos educacionais, psicológicos e socioculturais do envelhecimento, quando comparada a linha que estuda os aspectos biológicos e funcionais. Na produção analisada observa-se grande abrangência dos temas pesquisados, nos quais busca-se realizar a interface de saberes, métodos e compreensão dos resultados das investigações, que são também os objetivos para a continuidade das investigações dentro do enfoque interdisciplinar.
\end{abstract} DESCRITORES: Produção intelectual; Senescência; Multidisciplinaridade; Inovação.

\begin{abstract}
The integral approach to issues related to the elderly requires knowledge production and skilled labor. The establishment of graduate programs for gerontology and geriatrics area is the answer that educational institutions have offered. The goal of this article is to present what has been produced on Master on Sciences of Aging at São Judas Tadeu University, in São Paulo. The annual reports of Capes, between 2010 and 2014 , were the material on intelectual production, comprising complete articles and abstracts published in scientific journals and proceedings of national and international events on aging and related areas, was raised, tabulated and analyzed independently by two professors. A total of 74 publications in journals, 280 productions in national and 57 in international events, mostly held on co-authored by researchers from different areas and levels of expertise, including the involvement of external professional contribution to the institution, is distributed in a balanced way throughout this period of time. It is observed disparity in the number of projects and researchers involved when considered the two research lines proposed by the program, with greater participation of the one that deals with educational, psychological and socio-cultural compared to the line that studies the biological and functional aspects of aging. The analyzed production reveals a broad scope of topics researched, in which it was seek the knowledge of methods and understanding interface of research results, which are also the goals for the continuation of studies within the interdisciplinary focus.

KEYWORDS: Intelectual production; Senescence; Multidisciplinary: Innovation.
\end{abstract}

\footnotetext{
* Contribuições das autoras: Ana Lúcia Gatti, elaborou e redigiu os resumos, em português e inglês, redigiu a Introdução e Objetivos, fez a revisão do texto final; Carla Witter, elaborou e redigiu o Método, Resultados e Discussão, fez a revisão do texto final; Claudia Aranha Gil, redigiu a Introdução e Objetivos, elaborou e redigiu as Considerações Finais, fez a revisão do texto final; Rita Maria Monteiro Goulart, elaborou e redigiu os Resultados e Discussão, fez a adequação das citações e referencias nas normas Vancouver, fez a revisão do texto final.
} 


\section{INTRODUÇÃO}

A mudança na pirâmide etária no Brasil e no mundo, com uma proporção cada vez maior de pessoas com idades superiores a 60 anos, tem sido constatada e referida por organismos internacionais e nacionais de levantamento demográfico e de saúde, como a Organização das Nações Unidas ${ }^{1}$ e o Instituto Brasileiro de Geografia e Estatística ${ }^{2}$. O aumento da longevidade, que se traduz concretamente no envelhecimento populacional, não se manifesta, entretanto, de modo idêntico para todos os indivíduos, dado ser um fenômeno que envolve várias dimensões, além da cronológica. Assim, a abordagem do envelhecimento humano deve contemplar, além da questão etária, os aspectos biológicos, sociais, psicológicos e espirituais ${ }^{3}$.

Nesta perspectiva observa-se que ações voltadas a atender a complexidade das demandas nesse campo são muitas vezes baseadas em um modo de conhecimento estruturado em paradigmas e experiências disciplinares, fragmentárias ${ }^{4}$, que não contemplam a amplitude da demanda que se faz presente. Sendo assim, a abordagem integral torna-se necessária, nos diversos níveis de atenção à saúde, requerendo produção de conhecimento e trabalho em equipe, sem hierarquia, tal como tem sido refletido pela cultura institucional. ${ }^{5}$

Em um contexto mais amplo, autores como Rocha Filho $^{6}$ destacam que a fragmentação do conhecimento aplicada ao sistema educacional desde muito cedo traz inúmeros prejuízos para a formação científica necessária à educação de crianças e jovens. Entre eles, a carência do desenvolvimento precoce de um "espírito científico", capaz de questionar e romper dogmas, contribuindo assim para a formação de profissionais aptos a enfrentar os grandes desafios do nosso século, entre eles o envelhecimento populacional.

A Política Nacional do Idoso, ${ }^{7}$ anunciada pelo governo brasileiro em 1994, com o objetivo de assegurar os direitos sociais e atenção integral aos idosos, dispõe, entre outros aspectos, sobre a necessidade de preparo de profissionais especializados que estejam instrumentalizados não só para lidar diretamente com o idoso, mas que também possam contribuir para as discussões e debates sobre a temática. Assim, na área da saúde torna-se cada vez mais necessário que a formação ofertada tanto na graduação quanto na pós-graduação e educação permanente inclua conteúdos relativos à gerontologia ${ }^{8}$.

A capacitação de profissionais que atuem no campo da Gerontologia tem como base a articulação e integração de diversas áreas do conhecimento, ampliando assim também a possibilidade de integração das equipes de trabalho que possam atuar tanto na área da prevenção quanto na intervenção, considerando a heterogeneidade da população idosa ${ }^{9}$. A Gerontologia é, portanto, interdisciplinar em sua essência, na medida em que cria novas estruturas conceituais e acaba por romper as estruturas de origem, recombinando-as e sintetizando-as ${ }^{8}$.

A necessidade da promoção de qualidade de vida na velhice demanda, além da visão e atuação interdisciplinar, ações intersetoriais. Ao ampliar a questão, destaca-se a importância de políticas públicas intersetoriais para a população idosa em nosso país. Segundo Oliveira,Panhoca e Alves ${ }^{10}$, a implementação de tais políticas tem sido realizada de modo ainda muito setorizado, diferente de algumas ações na área da saúde que têm privilegiado a integração e o trabalho em rede, sendo necessária por parte dos gestores uma revisão da articulação e integração de tais ações na esfera pública.

Deste modo, o atendimento às diversas necessidades de cuidados, tanto no nível individual quanto governamental, tem de estar pautado na multi e interdisciplinaridade, com especial destaque para a última, na medida em que,segundo a Capes (2008 apud Alvarenga et al) ${ }^{11}$, esta permitiria avanço do conhecimento ao envolver intercâmbios tanto em relação a teorias quanto metodologias, o que visaria atender fenômenos mais complexos. Tal disposição tem sido verificada como propósito dos diversos programas de Pós-Graduação envolvidos com a questão do envelhecimento.

A constatação da necessidade de aprofundamento em relação ao envelhecimento e as várias interfaces que estão implicadas no conhecimento sobre os idosos tornou-se uma iniciativa concreta a partir do estabelecimento de programas de Pós-Graduação stricto sensu ao final do século passado. Assim surgiram, junto as universidades públicas e confessionais, os primeiros núcleos de produção de conhecimento sobre o envelhecimento.

O programa da Universidade de Campinas (Unicamp) em Gerontologia, aprovado pela Capes em 1997, apresentou, entre os primeiros candidatos e profissionais, o caráter da multi e interdisciplinar. No mesmo ano também surgiu o programa da Pontifícia Universidade Católica de São Paulo, com área de concentração na Gerontologia Social.

Após três anos, a Pontifícia Católica do Rio Grande do Sul ofereceu o primeiro curso de pós-graduação fora do estado de São Paulo: Gerontologia Biomédica. Também incluído na área interdisciplinar da Capes, assim como o da Unicamp, hoje oferece, além do nível mestrado, também o doutorado. 
A evidência da importância de tais programas pode ser referendada pelas diversas propostas acadêmicas, como a de Gerontologia da Universidade Católica de Brasília (DF), que iniciou suas atividades em 2003 e de Envelhecimento Humano da Universidade de Passo Fundo (RS), que passou a oferecer o curso em 2009. O Mestrado em Ciências do Envelhecimento da Universidade São Judas Tadeu (SP) teve início em 2010, sendo que no ano seguinte começou o programa de Saúde e Envelhecimento da Faculdade de Medicina de Marília (SP) e em 2013 o Mestrado de Gerontologia da Universidade Federal de Pernambuco.

Com uma proposta profissionalizante, o Mestrado oferecido em Mogi das Cruzes - SP, pelas Faculdades Educatie, em Psicogerontologia foi recomendado pela Capes em 2013 e passou a funcionar em 2014. Esse é o único mestrado profissional na área de gerontologia e que não está na área Interdisciplinar da Capes, foi recomendado pela área de Psicologia. Em 2015, iniciaram-se as atividades do Programa de Mestrado em Gerontologia oferecido pela EACH-USP Leste (SP), sendo que o programa foi recomendado pela Capes em 2014.

Também têm se observado um número crescente de programas de pós-graduação lato sensu sobre o tema envelhecimento, vinculados a instituições de ensino ou a centros de atendimento que recebem a população idosa, como hospitais. Como exemplo podem ser citados os cursos de lato sensu da Universidade Estadual do Rio de Janeiro, que foi constituído em torno da Universidade Aberta da Terceira Idade, os do Hospital Oswaldo Cruz e Hospital Israelita Albert Einstein, locais de referência de atendimento à saúde na cidade de São Paulo.

Em outubro de 2001 a Universidade São Judas Tadeu (USJT) iniciou seu primeiro movimento no sentido de oferecer à comunidade do entorno um projeto de extensão destinado exclusivamente à população idosa que recebeu o título "Projeto Sênior para a Vida Ativa". O projeto se caracteriza por compreender o processo de envelhecimento humano de forma interdisciplinar,integrando as áreas de Educação Física, Nutrição e Farmácia da USJT. É uma proposta multidisciplinar de caráter educacional, congregando esforços com o objetivo de educar os idosos que não tiveram amplo acesso à educação formal no país. Espera-se que a participação no projeto estimule a autonomia na velhice, considerando-se como aspecto essencial a manutenção de atitudes saudáveis pelos idosos, como a prática sistemática de atividades físicas, a adoção de dieta adequada, o constante autocuidado e o desenvolvimento de consciência crítica a respeito da cidadania do idoso de forma a contribuir para uma velhice bem sucedida ${ }^{12}$.
No entanto, não é apenas com o "Projeto Sênior para a Vida Ativa" que a vocação da Universidade São Judas Tadeu para o estudo, a pesquisa e o atendimento aos idosos é configurada. Questões relativas aos aspectos psicológicos do envelhecimento são investigadas, desde 2003, pelo Grupo de Pesquisa "Psicologia do Idoso" cadastrado no Diretório de Grupos de Pesquisa (DGP) do $\mathrm{CNPq}$, sendo apresentadas pesquisas em congressos no Brasil e no exterior, além de publicações científicas. Ainda, segundo Witter ${ }^{12}$, outro programa intitulado "Universidade Aberta à Maturidade" iniciou suas atividades em 1995, objetivando propiciar às pessoas de faixa etária mais elevada, além da aquisição de novos conhecimentos e aprimoramento dos conhecimentos já adquiridos, integração e bem estar psicossocial. Incluída entre as pioneiras do gênero no país e inicialmente denominada Universidade Aberta à Terceira Idade, em 2002, após reestruturação, passou a chamar-se Universidade Aberta à Maturidade, incluindo disciplinas necessárias às demandas sociais atuais, como uso dos recursos de informática, uso das redes sociais, conhecimento de geopolítica, direitos humanos e dos idosos, economia financeira, uso e costume dos diversos países, questões socioculturais entre outros temas de interesse.

O Programa de Pós-Graduação Stricto Sensu em Ciências do Envelhecimento (PGC) da Universidade São Judas Tadeu foi recomendado pelo Conselho Técnico Científico (CTC) da CAPES em 29/10/2009. Os Grupos de Pesquisa cadastrados no Diretório do CNPq (Conselho Nacional de Desenvolvimento Científico e Tecnológico) e certificados pela Universidade São Judas Tadeu (USJT), desde 2001 e 2003, foram sua origem. Tais grupos já vinham desenvolvendo pesquisas sobre o idoso e o processo de envelhecimento dentro de um enfoque multidisciplinar, que incluía diversas áreas do conhecimento, tais como: Psicologia, Educação, Ciências Biológicas, Educação Física, Nutrição e Letras, entre outras. Ainda hoje, a busca do conhecimento sobre os idosos e o processo de envelhecimento, em uma perspectiva ampla e totalizadora, é o objetivo perseguido pelo programa ${ }^{12}$.

A área de concentração definida pelo programa de mestrado foi Saúde, Educação e Qualidade de Vida, que comporta duas linhas de pesquisa: (1) Aspectos biológicos e funcionais do envelhecimento, da qual participam seis docentes e (2) Aspectos educacionais, psicológicos e socioculturais do envelhecimento, que conta com a participação de oito docentes. Sua abrangência tem permitido que alunos provenientes de diversas áreas busquem o conhecimento no programa e produzam, de modo interdisciplinar, seus trabalhos, em consonância com o objetivo amplo da proposta, que 
é desenvolver conhecimento sobre o envelhecimento abrangendo as interfaces de vários saberes, em uma perspectiva interdisciplinar que procura investigar aspectos relacionados com a vida biológica, psicológica, social e cultural no que se refere às questões da saúde, educação e qualidade de vida dos idosos ${ }^{12}$.

O programa busca produzir conhecimento sistemático sobre o idoso e o processo de envelhecimento, em função das características multideterminadas e multifacetadas do objeto de estudo. As áreas de formação básica dos docentes são diversificadas, a saber: Educação Física (6), Fisioterapia (2), Nutrição (3), Medicina (1) e Psicologia (3). Em 2015 foram contratados mais três professores, um com formação em Educação Física, um da área da Nutrição e um profissional com formação nas áreas de Educação Física e Fisioterapia. Os títulos de doutorado foram também em áreas diversas, tais como: Anatomia Funcional (1), Ciências (1), Ciências Morfofuncionais (1), Biologia Celular e Molecular (1); Ciências da Saúde (1), Fisiopatologia Experimental (1), Morfologia (1), Psicologia (4),Saúde Pública (2) e Educação Física (1), o que caracteriza um corpo docente com formação variada.A formação diversificada do corpo docente e a experiência, tanto profissional como acadêmica e científica, possibilita a discussão sobre o envelhecimento dentro do enfoque interdisciplinar e, portanto, a inserção do programa na Área Interdisciplinar da CAPES (Área 45) e na área temática da Câmara IV: Saúde e Biológicas. ${ }^{12}$

O PGCE procura formar profissionais com habilidades e competências dentro de uma perspectiva do envelhecimento como fenômeno de compreensão interdisciplinar que o torne capaz de agregar ao seu arcabouço teórico e a sua prática profissional uma visão de multidimensionalidade sob o escopo da saúde, educação e qualidade de vida. Ainda segundo Witter (2014) (12), espera-se que o egresso seja capaz de: (1) produzir conhecimento científico para a área de forma ética, tendo por base o compromisso social de melhoria da qualidade de vida da região e do Brasil; (2) atuar na pesquisa e na vida profissional com ética, competência e criticidade, quer individualmente quer em equipe multi ou interdisciplinar e (3) capacitar recursos humanos para o ensino-pesquisa nas instituições de ensino superior, de pesquisa, de empresas e de organizações que atuam na área da saúde e educação.

A estrutura curricular, composta por 24 disciplinas, foi organizada e elaborada pelos professores doutores, de acordo com a experiência acadêmica e profissional de cada um e de forma a contemplar os objetivos e o caráter interdisciplinar do programa de Mestrado. As cinco disciplinas obrigatórias propiciam a integração dos mestrandos, de diversas áreas do conhecimento, dentro do enfoque interdisciplinar com a discussão do objeto de estudo (envelhecimento) sob a perspectiva das diversas áreas de conhecimento que compõem as duas Linhas de Pesquisa ${ }^{12}$.

As disciplinas têm como foco o estudo do envelhecimento na perspectiva da interdisciplinaridade, com o objetivo de promover a reflexão e análise crítica de pesquisas e debates sobre as temáticas que envolvem o idoso e o processo de envelhecimento de forma que os Mestrandos compreendam a perspectiva das outras áreas de conhecimento e não se limitem à sua formação disciplinar. A proposta é criar e desenvolver uma forma de pensar, analisar e investigar o fenômeno como multideterminado e multifacetado, com a possibilidade de uma compreensão maior (para além da sua área de conhecimento) que permita ao discente criar um novo formato de investigação que favoreça o surgimento de projetos de pesquisa e extensão inovadores na área do envelhecimento ${ }^{12}$.

Outra característica da estrutura curricular que merece destaque são as Tutorias (I, II, III e IV), atividade obrigatória, com créditos, horários semanais e controle de frequência, nas quais são realizadas as atividades de orientação para a realização da dissertação de Mestrado pelo orientando, que recebe acompanhamento individual regular do orientador, o que aumenta a probabilidade de conclusão da dissertação e de artigos para publicação, dentro do prazo de dois anos estipulado pela CAPES. As Tutorias são realizadas em quatro módulos semestrais, de forma sequenciada, com o objetivo principal de garantir a orientação dos projetos de pesquisas dos Mestrandos durante os dois anos do curso. Essa atividade tem contribuído para o cumprimento dos prazos e das atividades, resultando na Defesa da Dissertação, na entrega de um manuscrito submetido a revista científica sobre a temática do trabalho de conclusão e no tempo médio de titulação de 24 até 30 meses $^{12}$.

O objetivo do presente relato é fazer uma apresentação do que foi produzido pelo programa de Mestrado em Ciências do Envelhecimento da Universidade São Judas Tadeu em seus cinco anos de funcionamento, a distribuição dos projetos pelos temas de investigação e a contribuição para o conhecimento na área.

\section{MÉTODO}

\section{Material}

Foram utilizadas as informações dos relatórios anuais da Capes, de 2010 até 2014, sendo levantadas as informações referentes à produção intelectual do corpo docente neste período. Foram analisadas as produções 
bibliográficas compreendendo artigo completo e resumo em anais (breve, expandido ou completo) publicados, respectivamente, em revistas científicas e anais de eventos nacionais e internacionais da área de envelhecimento e áreas correlatas. Levantou-se, também, os temas dos projetos conduzidos ao longo da existência do programa.

\section{Procedimento}

O levantamento e tabulação da produção científica e dos temas dos projetos do PGCE foram realizados por duas docentes permanentes do programa, de forma independente. Foi levantada a produção intelectual, de 2010 à 2014, sendo incluídos os artigos e resumos em anais de autoria individual e em coautoria. Os artigos foram classificados de acordo com a qualificação da Capes (Webqualis, 2015), que varia de A1 até "Sem Classificação". Os resumos foram categorizados de acordo com o tipo de evento: nacional ou internacional. Os temas dos projetos foram arrolados pelos títulos constantes nas bases do programa inseridas no relatório da CAPES na Plataforma Sucupira.

\section{RESULTADOS E DISCUSSÃO}

A Tabela 1 apresenta os artigos publicados durante os cinco anos de existência do programa, totalizando 74 estudos, na sua maioria em coautoria com um ou mais autores de áreas diferentes, sendo os trabalhos realizados pelo corpo docente permanente com a colaboração de participantes externos, doutores e mestres de outros programas ou egressos do PGCE. Nestes cinco anos, apenas uma publicação foi realizada de forma individual.Também, observa-se um equilíbrio na produção anual do programa, tendo ocorrido um leve aumento após a primeira avaliação trienal, de 2010-2012.
A produção em múltipla autoria é uma tendência valorizada nas diversas áreas de conhecimento, principalmente nos programas interdisciplinares e, consequentemente, na área de Gerontologia ${ }^{13}$. Espera-se que as redes colaborativas de pesquisadores promovam e aprofundem o conhecimento científico de uma determinada área e, em decorrência deste tipo de trabalho e ação, surjam pesquisas inovadoras sobre o objeto de estudo ${ }^{14}$.

A área interdisciplinar da Capes enfatiza a necessidade de investigações e pesquisas que envolvem diversas áreas do conhecimento no sentido de promover inovações em suas áreas de estudo. ${ }^{15} \mathrm{O}$ caráter interdisciplinar de objetos de estudo, como da gerontologia, propiciaria a criação de redes de colaboração que investiguem o idoso e o seu processo de envelhecimento com o objetivo não apenas de promover conhecimento sobre essa fase da vida, mas também de promover inovações científicas sobre o tema.

Em relação à qualificação dos artigos nos estratos do Qualis da Capes, observa-se a necessidade do corpo docente e coautores do PGCE diversificarem as publicações nos níveis das revistas, entre os estratos B2 e A1, pois há uma concentração de artigos publicados no estrato B3 que sofrem uma glosa de $20 \%$ na avaliação da Capes, prejudicando o desempenho do programa (CAPES, 2013a).${ }^{16}$ Essa concentração de publicações é significante, segundo o teste do qui quadrado $(\mathrm{p}<$ 0,0001). A quantidade de trabalhos apresentados durante os cinco anos de existência do programa mostra que houve um aumento na produção em 2013 e 2014, em função do resultado da avaliação trienal (CAPES, 2013b), ${ }^{17}$ entretanto o teste do qui quadrado revelou que não há diferença estatisticamente significante $(\mathrm{p}<0,0001)$ no aumento da produção, que ficou distribuída de forma homogênea nos últimos cinco anos.

Tabela 1. Distribuição de artigos publicados em periódicos segundo classificação Qualis da Capes (2010 - 2014)

\begin{tabular}{|c|c|c|c|c|c|c|c|c|c|c|c|c|c|}
\hline \multirow[t]{2}{*}{ Qualis } & \multicolumn{2}{|c|}{ A 1} & \multicolumn{2}{|c|}{ A 2} & \multicolumn{2}{|c|}{ B 1} & \multicolumn{2}{|c|}{ B 2} & \multicolumn{2}{|c|}{ B 3} & \multicolumn{2}{|c|}{ B 4-SC } & \multirow{2}{*}{ Tota } \\
\hline & Ind & Múlt & Ind & Múlt & Ind & Múlt & Ind & Múlt & Ind & Múlt & Ind & Múlt & \\
\hline 2010 & - & 1 & - & - & - & 3 & - & - & - & 5 & - & 1 & 10 \\
\hline 2011 & - & - & - & - & - & 3 & - & 2 & - & 8 & - & 2 & 15 \\
\hline 2012 & - & - & - & 4 & - & 5 & - & - & - & 3 & - & 1 & 13 \\
\hline 2013 & - & - & 1 & 1 & - & 5 & - & - & - & 9 & - & 3 & 19 \\
\hline 2014 & - & - & - & 5 & - & 6 & - & 1 & - & 3 & - & 2 & 17 \\
\hline Total & 0 & 1 & 1 & 10 & 0 & 22 & 0 & 3 & 0 & 28 & 0 & 9 & 74 \\
\hline
\end{tabular}

SC: Sem Classificação; Ind: individual; Múlt: Múltipla autoria. 
Brasil (2010) ${ }^{18}$, no Plano Nacional de PósGraduação - 2011 - 2020 (PNPG), são apresentados os parâmetros, evolução, diretrizes e tendências para o incremento da Pós-graduação no país, sendo enfatizado a importância da avaliação dos programas pela CAPES, considerado como exitoso, apesar de algumas distorções devido as características das diversas áreas de conhecimento. Neste sentido é enfatizado a importância da produção intelectual e científica dos programas e a criação da metodologia de avaliação dos periódicos pelo Sistema Qualis-CAPES e o seu constante aperfeiçoamento para atender as especificidades de cada área e servir de diretriz para as publicações dos programas nos estratos mais altos: A1 e A2. O PGCE tem trabalhado neste sentido, procurando atingir os estratos $\mathrm{A} 1$ e $\mathrm{A} 2$, havendo uma maior número de publicações no estrato $\mathrm{A} 2$, a partir de 2012, perfazendo o total de 11 artigos no período de 2012-2014.

A Tabela 2 mostra as publicações individuais e em múltipla autoria nos eventos nacionais e internacionais. Observa-se a prevalência de trabalhos de múltipla autoria, tanto nos eventos nacionais como internacionais, respectivamente, com o total de $280(74,66 \%)$ e $57(15,2 \%)$ produções em coautoria comparados as $21(5,6 \%)$ e 17 (4,53\%) publicações individuais. O teste do qui-quadrado confirma que essa diferença é significante ( $p<0,00001)$. Os trabalhos em múltipla autoria ou coautoria são valorizados no meio científico, pois podem servir como um parâmetro quanto à colaboração científica entre profissionais pesquisadores e instituições ${ }^{19}$.

O PGCE tem procurado atender essas exigências da Capes, na medida em que sua produção intelectual tem sido realizada em múltipla autoria, com doutores, mestre e alunos de diversas formações (CAPES, 2013a e 2013b). Entretanto é um grande desafio para os docentes realizar o entrelaçamento das duas linhas de pesquisa com investigações inovadoras dentro da perspectiva interdisciplinar. Neste sentido, o corpo docente tem trabalhado em conjunto promovendo discussões sobre os trabalhos realizados na Linha 1 e Linha 2. Os docentes da Linha 2 têm incentivado, também, a participação de alunos da graduação em eventos com apresentação e publicação de pesquisas sobre envelhecimento.

Além disso, procura-se realizar a inserção científica do programa nos Eventos Nacionais (80,26\%) e Internacionais $(19,73 \%)$, com a disseminação do conhecimento gerado no programa de Mestrado. Também, a participação nos eventos científicos colabora com a formação científica e profissional dos mestrandos e graduandos, na medida em que estabelecem contatos com outros pesquisadores de diversos níveis, áreas e instituições, o que permite a possibilidade de criação e da ampliação das redes colaborativas entre os pesquisadores.

Tabela 2. Distribuição da produção intelectual publicada em Anais de Eventos Nacionais e Internacionais (2010 - 2014).

\begin{tabular}{|c|c|c|c|c|c|c|c|c|c|c|}
\hline \multirow[b]{2}{*}{ Ano } & \multirow{2}{*}{$\begin{array}{r}\text { Tipo de } \\
\text { Produção }\end{array}$} & \multicolumn{2}{|c|}{ Trabalho Completo } & \multicolumn{2}{|c|}{ Resumo Expandido } & \multicolumn{2}{|c|}{ Resumo } & \multicolumn{2}{|c|}{ Subtotal } & \multirow{2}{*}{$\begin{array}{l}\text { Total } \\
\text { Geral }\end{array}$} \\
\hline & & Ind & Múlt & Ind & Múlt & Ind & Múlt & Ind & Múlt & \\
\hline \multicolumn{11}{|c|}{ Evento Nacional } \\
\hline 2010 & & 3 & 4 & 0 & 4 & 11 & 57 & 14 & 65 & 79 \\
\hline 2011 & & 0 & 2 & 0 & 1 & 2 & 76 & 2 & 79 & 81 \\
\hline 2012 & & 0 & 24 & 0 & 0 & 3 & 16 & 3 & 40 & 43 \\
\hline 2013 & & 0 & 4 & 2 & 11 & 0 & 43 & 2 & 58 & 60 \\
\hline 2014 & & 0 & 14 & 0 & 0 & 0 & 24 & 0 & 38 & 38 \\
\hline Subtotal & & 3 & 48 & 2 & 16 & 16 & 216 & 21 & 280 & 301 \\
\hline \multicolumn{11}{|c|}{ Evento Internacional } \\
\hline 2010 & & 0 & 0 & 0 & 4 & 0 & 11 & 0 & 15 & 15 \\
\hline 2011 & & 5 & 5 & 0 & 1 & 3 & 17 & 8 & 23 & 31 \\
\hline 2012 & & 1 & 0 & 0 & 0 & 7 & 11 & 8 & 11 & 19 \\
\hline 2013 & & 0 & 0 & 1 & 0 & 0 & 1 & 1 & 1 & 2 \\
\hline 2014 & & 0 & 0 & 0 & 5 & 0 & 2 & 0 & 7 & 7 \\
\hline Subtotal & & 6 & 5 & 1 & 10 & 10 & 42 & 17 & 57 & 74 \\
\hline Total Geral & & 9 & 53 & 3 & 26 & 26 & 258 & 38 & 337 & 375 \\
\hline
\end{tabular}

Ind: individual; Múlt: Múltipla autoria. 
Nota-se que houve uma redução na participação nos Eventos, após a avaliação trienal, pois houve uma mudança de estratégia na condução das atividades da pós-graduação para que houvesse uma maior concentração na produção intelectual do corpo docente e discente na elaboração dos manuscritos dos trabalhos apresentados nos Congressos para submeter as revistas entre os estratos B1 e A1. O PGCE tem se beneficiado com a participação sistemática do corpo docente e discente nos eventos científicos da área, na medida em que divulga a produção intelectual do programa, ao mesmo tempo em que permite uma maior visibilidade do próprio Mestrado em Ciências do Envelhecimento.

$\mathrm{Na}$ Tabela 3 são apresentados os projetos de pesquisa concluídos e em andamento em ambas as linhas e o número de participantes em cada projeto, incluindo docentes permanentes, participantes externos, discentes da graduação e da pós-graduação. É significante o maior número de participantes na linha 2, com o total de $974(84,69 \%)$ pesquisadores contra $176(15,30 \%)$ colaboradores da linha 1.

A maior participação concentra-se nos alunos de graduação da Linha 2, possivelmente pelas características dos projetos de pesquisas e as áreas de conhecimento, em particular, a Psicologia, a Nutrição e a Educação Física, que realizam trabalhos de iniciação científica e conclusão de curso na área de gerontologia com pesquisa de campo. Na Linha 1, muitos projetos são de modelo animal realizados com ratos Wistar albinos, que despertam menor interesse nos estudantes da área de humanas (Psicologia) e da Saúde (Nutrição e Educação Física), que preferem participar de pesquisas com seres humanos.

Entretanto, em ambas as linhas há constante participação de alunos da graduação $(\mathrm{N}=571$; $49,65 \%)$ e da pós-graduação $(\mathrm{N}=205 ; 17,82 \%)$, além dos professores $(\mathrm{N}=246 ; 21,39 \%)$ e participantes externos $(\mathrm{N}=128 ; 11,13 \%)$. $\mathrm{O}$ envolvimento dos alunos, de graduação e pós-graduação, e dos professores e pesquisadores externos revela a criação e existência de uma rede colaborativa em diversos níveis e áreas de conhecimento, com a parceria de diversas instituições na elaboração e produção científica e intelectual do programa de Mestrado. De acordo com Ralha, Carvalho e Guerra ${ }^{19}$ e Rodrigues $^{20}$ as redes colaborativas científicas podem ser definidas como processo de disponibilização de informação na qual os autores, pesquisadores, pessoas e grupos de pesquisa são os autores, considerados os "nós da rede", sendo que alguns são os vértices ou polos de conhecimento (professores/pesquisadores e instituições de prestígio) e outros são pontos menores na rede. Todos os atores são interligados pelas diversas vinculações acadêmicas, tais como a elaboração de pesquisas, projetos de pesquisa, participação em eventos, produção científica e intelectual conjunta, parcerias, enfim atividades de pesquisa, ensino e extensão que são denominadas com as conexões entre os atores da rede.

Tabela 3. Quantidade de Projetos de Pesquisa e de participantes por Linha de Pesquisa (2010 - 2014)

\begin{tabular}{|c|c|c|c|c|c|c|c|}
\hline \multirow[b]{2}{*}{ Ano } & \multicolumn{2}{|c|}{$\mathbf{N}$} & \multicolumn{2}{|c|}{$\mathbf{N}$} & \multicolumn{2}{|c|}{$\mathbf{N}$} & \multirow{2}{*}{$\begin{array}{c}\text { Total } \\
\text { Pesquisador }\end{array}$} \\
\hline & Ativo & Concluído & Prof. & $\begin{array}{c}\text { Participante } \\
\text { Externo }\end{array}$ & $\begin{array}{c}\text { Aluno } \\
\text { Graduação }\end{array}$ & $\begin{array}{c}\text { Aluno } \\
\text { Pós-Graduação }\end{array}$ & \\
\hline \multicolumn{8}{|c|}{ Linha 1 - Aspectos Biológicos e Funcionais do Envelhecimento } \\
\hline 2010 & 5 & 4 & 24 & 1 & 2 & 1 & 28 \\
\hline 2011 & 7 & 3 & 16 & 18 & 5 & 10 & 49 \\
\hline 2012 & 7 & 0 & 12 & 14 & 4 & 12 & 42 \\
\hline 2013 & 7 & 0 & 12 & 8 & 0 & 4 & 24 \\
\hline 2014 & 7 & 0 & 13 & 11 & 0 & 9 & 33 \\
\hline SubTotal & 7 & 7 & 77 & 52 & 11 & 36 & 176 \\
\hline \multicolumn{8}{|c|}{ Linha 2 - Aspectos Educacionais, psicológicos e socioculturais do Envelhecimento } \\
\hline 2010 & 11 & 1 & 22 & 0 & 72 & 19 & 113 \\
\hline 2011 & 17 & 3 & 32 & 7 & 149 & 27 & 215 \\
\hline 2012 & 22 & 1 & 37 & 13 & 124 & 36 & 210 \\
\hline 2013 & 22 & 1 & 39 & 24 & 105 & 35 & 203 \\
\hline 2014 & 22 & 1 & 39 & 32 & 110 & 52 & 233 \\
\hline SubTotal & 22 & 7 & 169 & 76 & 560 & 169 & 974 \\
\hline Total Geral & 29 & 14 & 246 & 128 & 571 & 205 & 1150 \\
\hline
\end{tabular}


Portanto, a criação e formação de pesquisadores por meio de redes colaborativas permitem o avanço e inovação nas áreas de conhecimento cujos objetos de estudo são complexos e exigem um enfoque interdisciplinar. O PGCE reconhece e tem incentivado a criação e solidificação das redes colaborativas científicas para a "formação de equipes maiores, aptas a enfrentar os desafios de complexidade crescente do desenvolvimento científico e tecnológico globalizado" 19 .

Em relação aos temas trabalhados nos Projetos de Pesquisa do Programa, é possível observar a interdisciplinaridade pela abrangência dos diferentes aspectos relacionados às dimensões envolvidas na vida do idoso exploradas entre 2010 e 2014. Os projetos compreendem desde questões sobre qualidade de vida, bem estar e espiritualidade até alterações fisiológicas próprias do envelhecimento ou acompanhadas por doenças crônicas não transmissíveis que necessitam de intervenção multidisciplinar (Tabela 4).

Observa-se na Tabela 4 maior concentração de projetos nos temas relacionados aos aspectos nutricionais, funcionais e fisiológicos $(\mathrm{N}=27)$, educação, arte, cultura e lazer $(\mathrm{N}=21)$, Qualidade de vida (idosos e cuidadores), bem estar, espiritualidade $(\mathrm{N}=16)$ e aspectos psicológicos e avaliação física $(\mathrm{N}=15)$ que juntos respondem por $56,8 \%$ dos trabalhos produzidos. Estes resultados mostram uma tendência de explorar aspectos relacionados com a promoção da saúde buscando desmistificar a relação envelhecimento-doença tão difundida no senso comum da sociedade. Houve uma menor concentração de temas que investigam doenças cardiovasculares - modelos humano e animal $(\mathrm{N}=18)$ que corresponde a $12,9 \%$ dos projetos que estão mais voltados para aspectos mais degenerativos do processo de envelhecimento.

A mudança de paradigma da produção de conhecimento disciplinar para interdisciplinar representa um desafio para os programas interdisciplinares da CAPES, pois é fundamental a existência de um arcabouço disciplinar sólido para dar qualidade a produção do programa. ${ }^{21}$ Entretanto, os pesquisadores (docentes e discentes) precisam colaborar com as diversas áreas de conhecimento, deixando a sua "zona de conforto", para compreender e colaborar com as outras áreas e, consequentemente, produzir um conhecimento interdisciplinar e inovador sobre o objeto de estudo na sua complexidade, como é o caso do idoso e o processo de envelhecimento. De acordo com Philippi Jr et al, ${ }^{21}$ a inovação "surge quando diferentes perspectivas se somam e revelam muito mais nuances da temática estudada do que a simples soma de perspectivas".

$\mathrm{Neri}^{22}$ destaca a importância do enfoque interdisciplinar para a compreensão da velhice em seus múltiplos aspectos determinantes para uma qualidade de vida satisfatória.Athayde, Bartels, Buschbacher e Seluchinesk ${ }^{23}$ discutem que a transdisciplinaridade e a aprendizagem colaborativa entre os diversos protagonistas de uma determinada área de estudos, como a gestão socioambiental na Amazônia, é fundamental para a produção e difusão de conhecimento científico na sociedade, como uma forma integradora dos diversos saberes. Rodrigues (2014) reforça a necessidade de criação, desenvolvimento e solidificação de redes colaborativas para o avanço científico e formação de pesquisadores, principalmente em localidades mais distantes de centros urbano, como os programas de pós-graduação na Amazônia.

Tabela 4. Distribuição de projetos segundo temas relacionados ao envelhecimento.

\begin{tabular}{|c|c|c|c|c|c|c|}
\hline Projetos - Temas & 2010 & 2011 & 2012 & 2013 & 2014 & Total \\
\hline Doenças Cardiovasculares - Modelos humano e animal & 4 & 5 & 2 & 4 & 3 & 18 \\
\hline Análise de produção científica & 3 & 2 & 2 & 2 & 2 & 11 \\
\hline Suplementação & 1 & 2 & 1 & - & - & 4 \\
\hline Imagem e Percepção Corporal & 1 & 1 & 1 & 1 & 1 & 5 \\
\hline Aspectos nutricionais, funcionais e fisiológicos & 4 & 7 & 5 & 5 & 6 & 27 \\
\hline Aspectos psicológicos e avaliação física & 1 & 1 & 6 & 2 & 5 & 15 \\
\hline Imunosenescência celular e molecular & 1 & 1 & - & - & - & 2 \\
\hline Educação, arte, cultura e lazer & 3 & 5 & 4 & 6 & 3 & 21 \\
\hline Qualidade de vida (idosos e cuidadores), bem estar, espiritualidade & 3 & 2 & 3 & 5 & 3 & 16 \\
\hline Atividade Física/exercícios & - & 2 & 2 & 2 & 3 & 9 \\
\hline $\begin{array}{l}\text { Alterações hemodinâmicas, músculo esqueléticas, morfofuncionais } \\
\text { associadas ao envelhecimento - Modelo animal }\end{array}$ & 1 & 2 & 4 & 1 & 3 & 11 \\
\hline Total & 22 & 30 & 30 & 28 & 29 & 139 \\
\hline
\end{tabular}


Em virtude do exposto, o PGCE tem buscado desenvolver projetos mais integradores, envolvendo os saberes das diversas disciplinas dentro de um enfoque interdisciplinar com a participação de colaboradores que formem e consolidem, com o desenvolvimento do programa, uma rede colaborativa de pesquisadores de diversos níveis e instituições. O desafio é grande, porém o programa de Ciências do Envelhecimento tem procurado melhorar os seus indicadores, não apenas quantitativos como os qualitativos para atender as últimas diretrizes do PNPG. ${ }^{18}$

\section{CONSIDERAÇÕES FINAIS}

As múltiplas demandas relacionadas ao crescente envelhecimento populacional têm representado um grande desafio na revisão e construção de paradigmas que contemplem as mais variadas questões relacionadas ao processo de envelhecimento na contemporaneidade. Nesse sentido, torna-se premente a formação e capacitação de recursos humanos, ou seja,profissionais aptos a atuar na área da gerontologia e que privilegiem a abordagem integral do idoso.

Frente a tal panorama, observa-se que o estabelecimento de Programas de Pós Graduação Stricto Sensu tem se intensificado, especialmente nas ultimas décadas,com a oferta de formação voltada às questões sobre o envelhecimento em várias regiões do país. No entanto é importante ressaltar que, ao lado do incremento da oferta, nota-se ainda a escassez de Programas na área da gerontologia, principalmente se levarmos em conta, entre outros aspectos, as amplas dimensões do nosso país, o acelerado crescimento da população idosa e o aumento da longevidade.

Nesse contexto, o programa de Mestrado Stricto Sensu da Universidade São Judas Tadeu tem em suas origens a necessidade de oferta de atendimento à comunidade idosa em seu entorno, pois se localiza no bairro da Mooca, região da cidade de São Paulo que possui um grande número de moradores dessa faixa etária. A partir da análise de sua produção intelectual,nota-se que há um aumento nos últimos dois anos, prevalecendo o número de trabalhos com múltiplas autorias e ampla temática, contemplando as várias dimensões da vida do idoso, estando assim coadunados com as bases que se espera dos programas interdisciplinares. Entre os temas destaca-se o número de trabalhos relacionados aos aspectos nutricionais, fisiológicos e funcionais, sendo considerado também o interesse por projetos relacionados a educação, arte e lazer, bem como sobre qualidade de vida, aspectos psicológicos e avaliação física. Verifica-se, em função da necessidade de aprimoramento do programa, a premência de diversificar as publicações, considerando a classificação dos artigos, segundo a Capes, aumentando a veiculação em revistas que estejam entre os estratos A1 e B1.

A necessidade da busca cada vez maior de conhecimento sobre o idoso e o processo de envelhecimento, abrangendo interfaces entre vários saberes em uma perspectiva interdisciplinar, tem motivado o corpo docente e discente a empreender esforços para a consolidação e excelência do programa. Busca-se, assim, um aprimoramento constante de todos os participantes, visando a construção e aplicação de conhecimento que se reverta em promoção de saúde e qualidade de vida à população que envelhece.

\section{REFERÊNCIAS}

1. WHO. Demographic Yearbook New York: WHO; 2013.

2. Portal Brasil. [Online]. Assistência ao Idoso. Brasilia; 2014 [cited 2014 outubro 10]. Available from: http://www.brasil. gov.br/saude/2014/10/dia-do-idoso-e-comemorado-nestaquarta-feira-1

3. Santos S. Concepções teórico-filosóficas sobre envelhecimento, velhice, idoso e enfermagem gerontogeriátrica. Revista Brasileira de Enfermagem. 2010; 63(6): 1035-9.

4. Scherer M, Pires D, Jean R. A construção da interdisciplinaridade no trabalho da Equipe de Saúde da Família. Ciência \& Saúde Coletiva. 2013;18(11):3203-12.

5. Besse M, Cecilio LCO, Lemos ND. A Equipe Multiprofissional em Gerontologia e a Produção do Cuidado: um estudo de caso. Revista Kairós Gerontologia. 2014;17 (2):205-22.

6. Rocha Filho J, Basso N, Borges R. Transdiciplinaridade: a natureza íntima da educação científica Porto Alegre: EDIPUCRS; 2007.

7. Brasil. Ministério da Previdência e Assistência Social. Plano de ação governamental integrado para o desenvolvimento da Política Nacional do Idoso. 1996.

8. Motta L, Aguiar A. Novas competências em saúde e o envelhecimento populacional brasileiro: integralidade, interdisciplinaridade e intersetorialidade. Ciência \& Saúde Coletiva. 2007;12(2):363-72.

9. Falcão D, Cachioni M, Yassuda M. Contribuições da Psicologia a Gerontologia: reflexões sobre ensino, pesquisa e extensão. Revista Kairós. 2009;4:43-58.

10. Oliveira A, Ramos O, Panhoca I, Alves V. A intersetorialidade nas políticas públicas para o envelhecimento no Brasil. Revista Kairós Gerontologia. 2014;17(2):91-103.

11. Alvarenga A, Philippi Jr A, Sommerman A, Alvares A, Fernandes V. Histórico, fundamentos fisosóficos e teóricometodológicos da interdisciplinaridade. In: Philippi Jr A, Silva Neto A. Interdisciplinaridade em ciência, tecnologia e inovação. Barueri: Manole; 2011. p. 3-68.

12. Witter C. Relatório do Programa de Pós-Graduação em Ciências do Envelhecimento. Plataforma Sucupira, CAPES. São Paulo: Universidade São Judas Tadeu, Departamento de Ciências do Envelhecimento; 2014.

13. Witter C, Buriti M, Witter G. Envelhecimento e contingências de vida. Campinas: Alínea; 2011. 
14. Población D, Mugnaini R, Ramos L. Redes sociais e colaborativas em informação científica. São Paulo: Angellara; 2009.

15. Philippi Jr A, Silva Neto A. Interdisciplinaridade em ciência, tecnologia e inovação. Barueri: Manole; 2011.

16. CAPES. Documento de área-2013. Brasília, Coordenação de aperfeiçoamento de pessoal de nivel superior. Diretoria de avaliação. Avaliação Trienal-2013; 2013a.

17. CAPES. Relatório de avaliação 2010-2012 - Trienal-2013. Brasília, Coordenação de aperfeiçoamento de Pessoal de Nível Superior, Diretoria de Avaliação. Avaliação Trienal-2013; 2013b.

18. Brasil. Ministério da Educação. Coordenação de Aperfeiçoamento de Pessoal de Nível Superior. Plano Nacional de Pós-Graduação - PNPG 2011-2020. CAPES. 2010.

19. Ralha CG, Carvalho DBB, Guerra GN. ObservUnB: Observatório da Rede de Colaboração Científica da
Universidade de Brasília. Revista Brasileira de PósGraduação - RBPG. 2013;10(22):1133-60.

20. Rodrigues RO. Pós-graduação na Amazônia: o desafio de formar (em) redes. Revista Brasileira de Pós-Graduação RBPG. 2014;10(21):19-45.

21. Philippi Jr A, Sobral MC, Fenandes V, Sampaio CAC. Desenvolvimento sustentável, interdisciplinaridade e Ciências Ambientais. Revista Brasileira de Pós-Graduação - RBPG. 2013;10(21):509-33.

22. Neri AL. Qualidade de vida na velhice: enfoque multidisciplinar. Campinas: Alínea; 2007.

23. Athayde S, Bartels WL, Buschbacher R, Seluchinesk RDR. Aprendizagem colaborativa, transdisciplinaridade e gestão sociomabiental na Amazônia: abordagens para a construção de conhecimento entre academia e sociedade. Revista Brasileira de Pós-Graduação - RBPG. 2013;10(21): 729-56. 